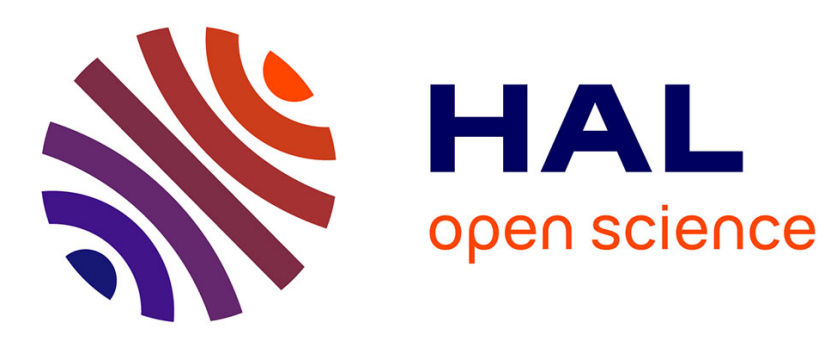

\title{
Reliability model of a component equipped with PHM capabilities
}

\author{
Michele Compare, Luca Bellani, Enrico Zio
}

\section{To cite this version:}

Michele Compare, Luca Bellani, Enrico Zio. Reliability model of a component equipped with PHM capabilities. Reliability Engineering and System Safety, 2017, 168, pp.4-11. 10.1016/j.ress.2017.05.024 . hal-01652191

\section{HAL Id: hal-01652191 \\ https://hal.science/hal-01652191}

Submitted on 30 Nov 2017

HAL is a multi-disciplinary open access archive for the deposit and dissemination of scientific research documents, whether they are published or not. The documents may come from teaching and research institutions in France or abroad, or from public or private research centers.
L'archive ouverte pluridisciplinaire HAL, est destinée au dépôt et à la diffusion de documents scientifiques de niveau recherche, publiés ou non, émanant des établissements d'enseignement et de recherche français ou étrangers, des laboratoires publics ou privés. 


\title{
Reliability model of a component equipped with PHM capabilities
}

\author{
Michele Compare ${ }^{\mathrm{a}, \mathrm{b}}$, Luca Bellani ${ }^{\mathrm{a}}$, Enrico Zio ${ }^{\mathrm{a}, \mathrm{b}, \mathrm{c}, *}$ \\ ${ }^{a}$ Energy Department, Politecnico di Milano, Italy \\ ${ }^{\mathrm{b}}$ Aramis S.r.l., Italy \\ ${ }^{\mathrm{c}}$ Chair on Systems Science and the Energetic challenge, Foundation Electricité de France at CentraleSupélec, France
}

\section{A R T I C L E I N F O}

\section{Keywords:}

PHM metrics

Reliability

Particle Filtering

Monte Carlo Simulation

\begin{abstract}
A B S T R A C T
We propose an analytic, time-variant model that conservatively evaluates the increase in reliability achievable when a component is equipped with a Prognostics and Health Management system of known performance metrics. The reliability model builds on metrics of literature and is applicable to different industrial contexts. A simulated case study concerning crack propagation in a mechanical component is considered to validate the proposed model.
\end{abstract}

(C) 2017 Elsevier Ltd. All rights reserved.

\section{Introduction}

In the last decade, Prognostics and Health Management (PHM) has often been proposed as an effective technology to respond to the reliability challenges posed by the modern safety-critical components and systems (e.g., nuclear power plants, oil\&gas assets, etc.), in which failures can result not only in significant costs, but also in life-threatening consequences such as explosions and natural disasters.

PHM allows in principle monitoring the system health condition, predicting its Remaining Useful Life (RUL) and, ultimately, preventing catastrophic failures [1-5]. However, in practice it is important to know which are the reliability and availability of a component or system. In this respect, to the authors' best knowledge a modeling framework that allows translating the PHM contribution into the component or system reliability is still lacking.

A few works have attempted to evaluate the influence of PHM on system Life Cycle Cost (LCC, [6-11]), looking at the economic benefits of PHM in terms of increase of component or system availability. On the other hand, for safety-critical applications PHM is expected to mainly increase the component or system reliability (rather than availability). PHM helps avoiding over-estimations of the actual component RUL, which may lead to accidents with possible consequences on the asset, the environment and the public.

To evaluate the added value of the PHM technology on system reliability, it is necessary to characterize the performance of the PHM adopted. In this respect, a variety of performance metrics and indicators have been introduced for detection (i.e., the recognition of a deviation from the normal operating conditions causing such deviation, e.g., $[8,12]$ ), diagnostics (i.e., the characterization of the abnormal state, e.g., [13]) and prognostics, (i.e., the prediction of the evolution of the abnormal state up to failure, e.g., $[2,14,15])$. The original contribution of this work is to propose a general modeling and decision framework for linking PHM metrics of literature to the component reliability. This framework also allows accounting for the decision criterion adopted for maintenance (overhaul), which heavily depends on the risk attitude of the decision maker.

The proposed reliability model is validated by way of a simulated case study concerning the crack propagation in a mechanical component, which requires to estimate the values of the relevant PHM metrics.

Although various definitions of performance metrics exist in the PHM literature, a detailed procedure to estimate their values is still lacking, apart from a few metrics such as the MTTF [16]. For this, a further original contribution of our work is the Monte Carlo (MC) procedure proposed to estimate the performance metrics encoded in the developed reliability model.

The remainder of the paper is organized as follows: Section 2 briefly introduces the general framework; in Section 3, the impact of a PHM tool on system reliability is modeled; Section 4 illustrates a simulated case study concerning the crack propagation in a mechanical component; Section 5 validates the developed model by way of the simulated case study; Section 6 concludes the work.

\section{Modeling framework}

We consider a degrading component, whose degradation state is monitored every $\Delta t$ units of time with respect to a continuous indicator variable (Fig. 1). The degradation process is stochastic for the degradation state and two thresholds are considered: the detection threshold, which mainly depends on the characteristics of the instrument used for monitoring the degradation variable (for example, considering that the instrument is not capable of detecting the degradation state for values

\footnotetext{
* Corresponding author at: Energy Department, Politecnico di Milano, Italy.

E-mail address: enrico.zio@polimi.it (E. Zio).
} 


\begin{tabular}{|c|c|}
\hline \multicolumn{2}{|c|}{ Nomenclature } \\
\hline$\lambda$ & $\begin{array}{l}\text { Time window modifier, such that } t_{\lambda}=T_{p r}+\lambda\left(T_{f}-T_{p r}\right) \\
\lambda \in[0,1]\end{array}$ \\
\hline$\lambda^{*}$ & $\begin{array}{l}\text { Time from which the values of the performance metrics } \\
\text { are estimated }\end{array}$ \\
\hline$T_{d}$ & $\begin{array}{l}\text { Time instant at which the system reaches the detection } \\
\text { threshold }\end{array}$ \\
\hline$T_{f}$ & $\begin{array}{l}\text { Time instant at which the system reaches the failure } \\
\text { threshold }\end{array}$ \\
\hline$T_{\phi}$ & Length of the time interval $T_{f}-T_{d}$ \\
\hline$f_{T_{d}}$ & pdf of time $T_{d}$ \\
\hline$f_{T_{\phi}}$ & pdf of $T_{\phi}$ \\
\hline$f_{T_{f}}^{\varphi}$ & pdf of $T_{f}$ \\
\hline$\Delta t$ & $\begin{array}{l}\text { Time interval between two successive Remaining Use- } \\
\text { ful Life (RUL) predictions }\end{array}$ \\
\hline DTD & Detection Time Delay, $T_{p r}-T_{d}$ \\
\hline$f_{D T D}$ & probability density function (pdf) of DTD \\
\hline$P_{\lambda}^{\alpha}$ & $\alpha-\lambda$ performance \\
\hline$\lfloor x\rfloor$ & Integer part of $x$; that is, $n \leq x<n+1, x \in \mathbb{R}, n \in \mathbb{N}$ \\
\hline$N$ & Number of maximum RUL predictions before failure \\
\hline$k^{*}$ & $\begin{array}{l}\text { Index of the first time channel at which the decision to } \\
\text { remove the system from operation can be taken }\end{array}$ \\
\hline$h^{*}$ & $\begin{array}{l}\text { Index of the first time channel at which a missing alarm } \\
\text { is risky }\end{array}$ \\
\hline$R_{\lambda}$ & Uncertain predicted RUL at time indicated by $\lambda$ \\
\hline $\mathrm{Y}_{\lambda}$ & $\begin{array}{l}\text { Point summarizing the uncertainty in } R_{\lambda} \text { (e.g., mean, } \\
\text { median, } 10 \text { th percentile, etc.) }\end{array}$ \\
\hline$R U L_{\lambda}^{*}$ & Actual RUL at the time indicated by $\lambda$ \\
\hline$T_{p r}$ & Time of the first RUL prediction \\
\hline$F P$ & False positives \\
\hline FN & False negatives \\
\hline$m$ & Empirical estimate of metric $M$ \\
\hline$f_{R_{\lambda}}$ & $\begin{array}{l}\text { pdf of the predicted RUL at the time window indicated } \\
\text { by } \lambda\end{array}$ \\
\hline $\mathcal{N}\left(\mu, \sigma^{2}\right)$ & Normal distribution with mean $\mu$ and variance $\sigma^{2}$ \\
\hline $\mathcal{V}(a, b)$ & Uniform distribution between $a$ and $b$ \\
\hline
\end{tabular}

below such threshold), and the failure threshold, above which the component does not function any more or, more practically, must be maintained or replaced for avoiding a catastrophic failure.

The uncertainty in the time instant $T_{d}$ at which the component reaches the first threshold is described by the probability density function (pdf) $f_{T_{d}}$. If no action is taken, the component continues its degrading up to failure occurring at time $T_{f}$; its uncertainty is described by pdf $f_{T_{f}}$. Finally, we also consider the random variable $T_{\phi}=T_{f}-T_{d}$, whose pdf is $f_{T_{\phi}}$

Realistically, it is assumed that detection is not perfect. Thus, metrics of literature are exploited to characterize the detection performance. In this respect, the following two are widely used in practice: false positive probability (i.e., the probability of triggering undue alarms) and false negative probability (i.e., the probability of missing alarm when required) [8]). In addition, Detection Time Delay (DTD, [12]) is a detection metric which measures the interval from the time when the detectable degradation state is reached by the component up to its detection. We use this performance metric, due to two main reasons: on one hand, DTD is viewed as a false negative indicator which depends on time (i.e., alarms are missing up to DTD); on the other hand, the DTD values are dependent on the detection algorithm settings, which can be adjusted so that the false positive probability is negligible in the inital part of the component life [12]. This way, the model development is simplified. To be realistic, we assume that DTD is affected by uncertainty, whose pdf is $f_{D T D}(\delta)$.

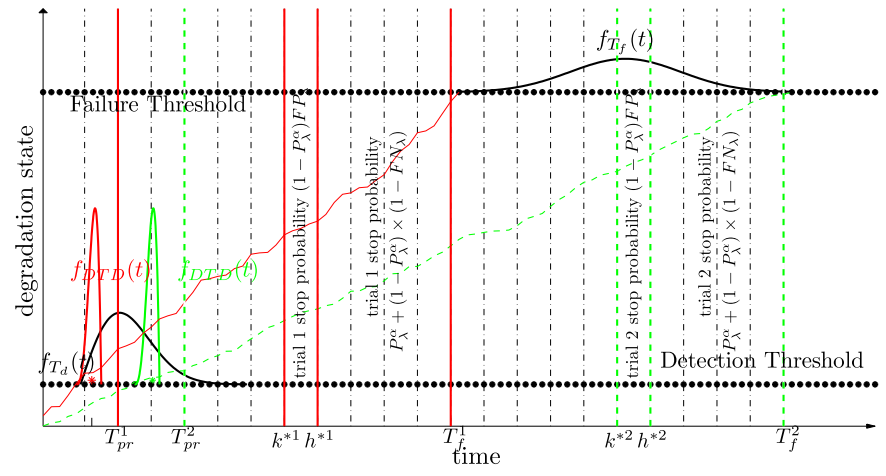

Fig. 1. Model setting description; $h=4, \alpha=0.1, N^{1}=11$ and $N^{2}=19$.

In this setting, the PHM system starts to predict the RUL at time $T_{p r}=\left(\left\lfloor\frac{T_{d}+D T D}{\Delta t}\right\rfloor+1\right) \Delta t$, where $\lfloor\bigcirc\rfloor$ indicates the integer part of its argument. The number of predictions that the PHM can perform before failure is $N=\left\lfloor\frac{T_{f}-T_{p r}}{\Delta t}\right\rfloor$. From now on, it is assumed that the system actually fails at time $T_{p r}+N \Delta t$, instead of $T_{f}$; the smaller $\Delta t$, the smaller the approximation.

Notice that we have assumed, for simplicity, that the considered component is affected by a single failure mode, so that we do not have the need of tackling the issue of embedding diagnostic metrics into the reliability model, and of considering all scenarios originating from decisions based on erroneous diagnoses of the failure mode. Such diagnostic issue is left for the future research work.

Finally, notice also that, in practice, both detection and failure thresholds may not be easily determined. For example, in helicopter applications, PHM systems (also called Health and Usage Monitoring System, HUMS) are mainly based on vibration monitoring to infer the equipment health [17-19]; thus, there is no simple way to define a threshold directly related to failure. Similar challenges are encountered in the packaging industry, where the failure conditions of components may not be precisely known [20]. Nonetheless, the approach proposed in the present work applies to any system, provided that some criterion to define the thresholds exists. The definition of such criterion is out of the scope of this work, where we assume that the Decision Maker (DM) has already defined a threshold coherent with his/her objective.

\section{Reliability model}

In this Section, we illustrate the mathematical model developed to evaluate the increase in system reliability brought by a PHM system.

We assume that the PHM-equipped component is stopped when the $(100-\beta)$ th percentile (e.g., $100-90=10$ th) of the currently predicted RUL pdf is smaller than $h \cdot \Delta t$ : the larger the value of $\beta$, the smaller the value of the predicted RUL percentile, the more risk-averse the decision. Similarly, the larger the value of $h$, the more cautious the decision maker.

To set $h$ and $\beta$ in real industrial applications, it should be kept in mind that the value of $h$ strongly depends on the time required to safely remove the component from operation (e.g., time required for system shutdown), whereas $\beta$ relates to the risk associated to the failure (e.g., $\beta=5$ is a very conservative value, suitable for safety critical application). To help the DM to set $h$ and $\beta$ we can use the proposed reliability model in a 'reverse' way, to find the combinations of values of $h$ and $\beta$ that allow meeting the system reliability requirements, also taking into account the considered PHM performance values. Furthermore, we can evaluate the sensitivity of the component reliability value to the selected applicable values of $h$ and $\beta$, to find the settings which are less sensitive to the possible variability of the metrics due to the uncertainty in their estimations.

To evaluate the probability of removing the system from operation before failure, we need to consider a time-variant prognostic perfor- 


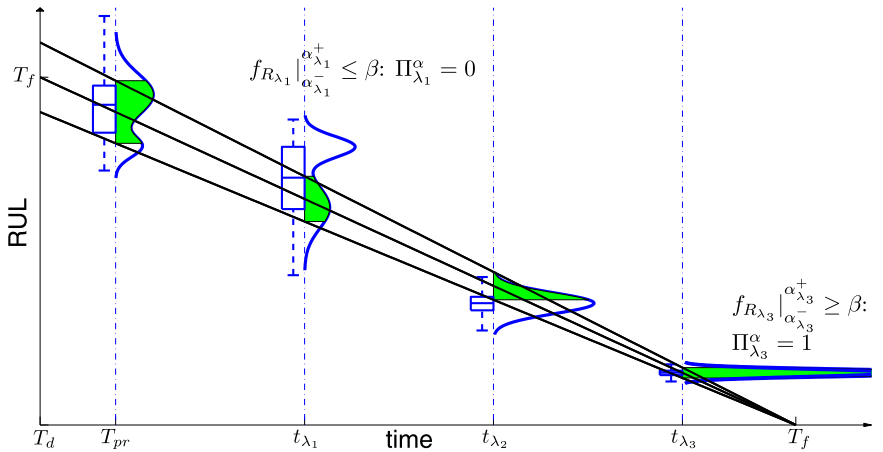

Fig. 2. $P_{\lambda}^{\alpha}$ description.

mance index and link it to the probability of being in stopping conditions.

Among the prognostic metrics available in the literature $[14,15]$, the most suitable is the $\alpha-\lambda$ performance index, $P_{\lambda}^{\alpha}$, which is a time-variant accuracy indicator ranging in $[0,1]$; this allows us to give $P_{\lambda}^{\alpha}$ a probabilistic interpretation. Various definitions of $P_{\lambda}^{\alpha}$ have been proposed in the literature $[14,15]$, referring to either point-wise or pdf RUL predictions. In this work, we give the following definition, derived from [14] (Fig. 2).

Consider the indicator variable:

$\Pi_{\lambda}^{\alpha}= \begin{cases}1, & \text { if }\left.f_{R_{\lambda}}\right|_{\alpha_{\lambda}^{-}} ^{\alpha_{\lambda}^{+}} \geq \beta \\ 0, & \text { else }\end{cases}$

where $f_{R_{\lambda}}$ is the pdf of the RUL $R_{\lambda}$ predicted at time $t_{\lambda}=T_{p r}+\lambda\left(T_{f}-\right.$ $\left.T_{p r}\right), \lambda \in[0,1]$, whereas $\alpha$ is a user-defined parameter which indicates the required tolerance around the value of $R U L^{*}$ (e.g., $\left.\alpha \in[0.05,0.2]\right)$.

Then, $P_{\lambda}^{\alpha}$ is the mean value of $\Pi_{\lambda}^{\alpha}$, i.e., $P_{\lambda}^{\alpha}=\mathbb{E}\left[\Pi_{\lambda}^{\alpha}\right]$.

Namely, during the test campaign of the algorithm, in which the value of the prognostic performance metrics are computed, the algorithm is run on the working system an as large as possible number of times. Then, at any trial, $\Pi_{\lambda}^{\alpha}$ is set to 1 if the RUL pdf predicted at $t_{\lambda}$ has an area larger than $\beta$ between $\alpha_{\lambda}^{-}=(1-\alpha) R U L_{\lambda}^{*}$ and $\alpha_{\lambda}^{+}=(1+\alpha) R U L_{\lambda}^{*}$, being $R U L_{\lambda}^{*}$ the actual RUL at time $t_{\lambda}$, i.e., the time up to reaching the failure threshold or the threshold above which a maintenance action must be performed, depending on the application (Fig. 2). The RUL* value is exactly known at the end of every trial.

$P_{\lambda}^{\alpha}$ is, then, practically given by the estimate $p_{\lambda}^{\alpha}$, which is calculated by averaging the values $\Pi_{\lambda}^{\alpha}$ gathered from different trials of the PHM tool at as many as possible instants $t_{\lambda}$. The larger the value of $P_{\lambda}^{\alpha}$, the better the PHM system prediction capability. For more details on $p_{\lambda}^{\alpha}$ computation, see Section 5.2.

Notice that when $\Pi_{\lambda}^{\alpha}=0$, no inference can be made about the value of the uncertain $R U L$ prediction: one only knows that the area overlapping $\left[\alpha_{\lambda}^{-}, \alpha_{\lambda}^{+}\right]$is smaller than $\beta$, with no further information about either the actual extent of this overlapping or the portion of probability mass located below $\alpha_{\lambda}^{-}$, above $\alpha_{\lambda}^{+}$or in an in-between position.

Notice also that when $\Pi_{\lambda}^{\alpha}=1$, then the interval $\left[(1-\alpha) R U L_{\lambda}^{*},(1+\right.$ $\left.\alpha) R U L_{\lambda}^{*}\right]$ is the 2 -sided $\beta$ confidence interval of the failure time predicted at time $t_{\lambda}$. However, for the prediction metrics to be applicable for supporting risk-averse decision making, we need to refer to an upper bound of the probability of over-estimating the RUL (i.e., of not stopping the component), rather than to a 2-sided confidence interval. To cope with this situation, we combine $P_{\lambda}^{\alpha}$ with the false positive and false negative metrics [15], which are time-variant indexes defined as, respectively:

$F N_{\lambda}=\mathbb{E}\left[\Phi N_{\lambda}\right], \Phi N_{\lambda}= \begin{cases}1, & \text { if } \Upsilon_{\lambda}-R U L_{\lambda}^{*}>d_{\lambda}^{\text {threshold }} \\ 0, & \text { else }\end{cases}$

$F P_{\lambda}=\mathbb{E}\left[\Phi P_{\lambda}\right], \Phi P_{\lambda}= \begin{cases}1, & \text { if } \Upsilon_{\lambda}-R U L_{\lambda}^{*}<-d_{\lambda}^{\text {threshold }} \\ 0, & \text { else }\end{cases}$

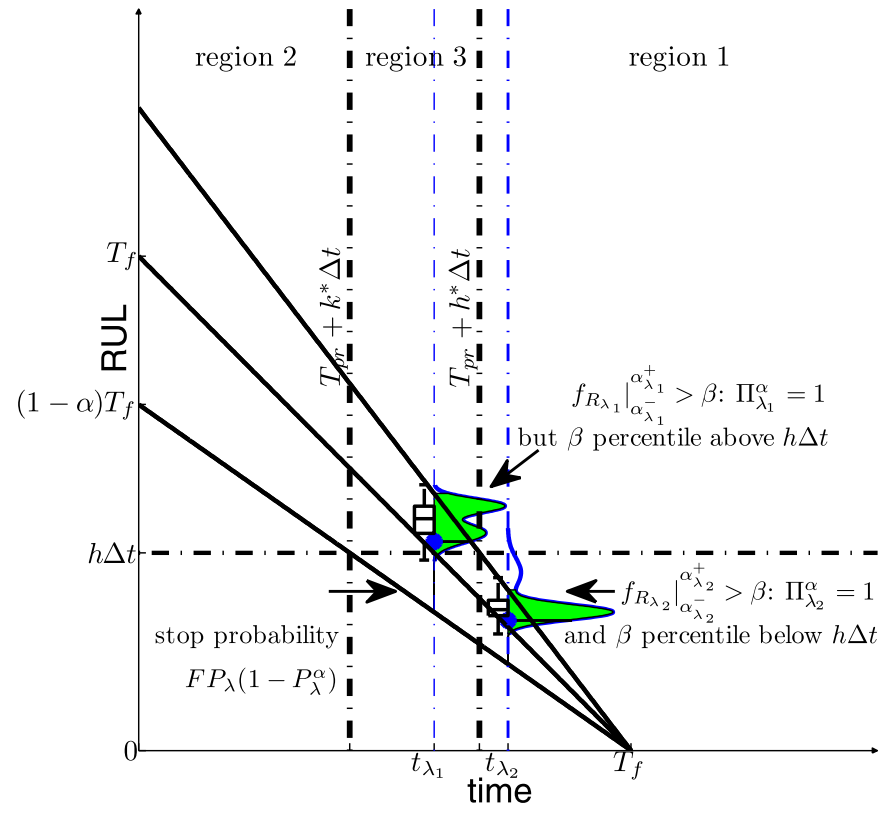

Fig. 3. Regions partitioning the time horizon and examples of possible RUL predictions.

where $\mathrm{Y}_{\lambda}$ is a point estimate of the predicted $R U L$ distribution (e.g., the mean, the median or any other percentile of $R_{\lambda}$, etc.) and $d_{\lambda}^{\text {thresold }}$ is a user-defined threshold value, which depends on the PHM application. Proceeding exactly in the same way as that of $p_{\lambda}^{\alpha}$, we will consider the estimates $f n_{\lambda}$ and $f p_{\lambda}$ of $F N_{\lambda}$ and $F P_{\lambda}$, respectively, which are given by the corresponding empirical averages of $\Phi N_{\lambda}$ and $\Phi P_{\lambda}$ over the available number of test trials, achieved through an algorithm test campaign. As mentioned above, notice that when performing a PHM test, $R U L^{*}$ is exactly known at the end of every trial. This value is, then, used to estimate $\Phi N_{\lambda}, \Phi P_{\lambda}$ and the other variables of the model, as shown in Section 5.2.

In our setting, $\mathrm{Y}_{\lambda}$ is the $(1-\beta)$ th percentile of $f_{R_{\lambda}}$ and $d_{\lambda}^{\text {threshold }}=$ $\alpha \times R U L_{\lambda}^{*}$. Then, $F P_{\lambda}$ measures the average portion of times in which $\mathrm{Y}_{\lambda}$ is below $\alpha_{\lambda}^{-}=(1-\alpha) R U L_{\lambda}^{*}$ and, thus, it becomes an indicator of how much conservative our PHM predictions are at time $t_{\lambda}$. Similarly, $F N_{\lambda}$ indicates the riskiness of the PHM algorithm.

Based on these considerations, we can build the reliability model of a PHM-equipped component with estimated values $p_{\lambda}^{\alpha}, f n_{\lambda}, f p_{\lambda}$ of metrics $P_{\lambda}^{\alpha}, F N_{\lambda}, F P_{\lambda}$, respectively. To do this, we divide the time horizon into three regions (Fig. 3):

1. The region in proximity of failure, which is defined by the time indexes $k \geq N-h$ such that $(1+\alpha) R U L_{\lambda}^{*} \leq h \Delta t$, where $R U L_{\lambda}^{*}=$ $(N-h) \Delta t$. This is the same as $k \geq h^{*}$, where $h^{*}=\left\lfloor N-\frac{h}{1+\alpha}\right\rfloor$. Geometrically, this region corresponds to time values on the right of the intersection between the error upper bound line $(1+\alpha) R U L_{\lambda}^{*}$ and the horizontal line positioned at $R U L=h \Delta t$ (Fig. 3).

2. The safe region, which is indicated by time instants $k<k^{*}$, where $k^{*}$ geometrically corresponds to the prediction most proximal to the intersection between the prediction error lower bound line $(1-\alpha) R U L_{\lambda}^{*}$ and the horizontal line at $R U L=h \Delta t$ (Fig. 3).

3. The in-between region, identified by $k^{*} \leq k<h^{*}$.

With respect to region 1 , we can note that to have a failure, the alarm is required to be missing $h^{*}$ consecutive times. Now, if $\Pi_{\lambda}^{\alpha}=1$, then the alarm is triggered and the component failure is avoided. On the contrary, if $\Pi_{\lambda}^{\alpha}=0$, the necessary condition to not activate the alarm is that the RUL is over-estimated. This situation occurs with probability $\left(1-P_{\lambda}^{\alpha}\right) F N_{\lambda} \simeq\left(1-p_{\lambda}^{\alpha}\right) f n_{\lambda}$. We assume that this probability value also describes the uncertainty in having missing alarms; this is a very conservative assumptions: the closer the current time to failure, the larger 
the over-estimation error required to not trigger the alarm (predictions must be above the $h \Delta t$ threshold (see Fig. 3)).

With respect to the safe region, we first note that whichever the value of $\Pi_{\lambda}^{\alpha}$, an over-estimation of $R U L_{\lambda}^{*}$ leads to not stopping the system before failure. This does not entail any risk of missing stops. On the contrary, an under-estimation of the RUL could lead to component stop. In the risk-averse setting we are dealing with, the anticipated maintenance is beneficial for system reliability, as it avoids component failure. For this, we conservatively assume that in this left-most region the PHM system never stops the component.

Finally, with respect to the in-between time horizon region, to rigorously derive the probability of not stopping the system, we have to give account to the fact that some extreme cases may occur, where even if $\Pi_{\lambda}^{\alpha}=1$, the $1-\beta$ probability mass and, thus, the $(1-\beta)$ th percentile, is positioned above $h \Delta t$. For example, Fig. 3 shows the situation where $t_{\lambda_{1}}=(N-h) \Delta t$ and all the $\beta$ mass is concentrated between $R U L_{\lambda}^{*}=h \Delta t$ and $\alpha_{\lambda}^{+}$. In this case, PHM will not advice to stop the component at $t_{\lambda_{1}}$. Thus, we conservatively assume that in this region the component does not undergo a maintenance action as long as $\Pi_{\lambda}^{\alpha}=1$.

On the contrary, when $\Pi_{\lambda}^{\alpha}=0$, which occurs with probability (1 $\left.P_{\lambda}^{\alpha}\right)$, the following three possible situations can occur:

- The $(1-\beta)$ th percentile, $\mathrm{Y}_{\lambda}$, is smaller than $(1-\alpha) R U L_{\lambda}^{*}$. In this situation, which occurs with probability $\left(1-P_{\lambda}^{\alpha}\right) F P_{\lambda}$, even if we conservatively assume that the $(1-\beta)$ th percentile takes the largest possible value (i.e., $\left.\Upsilon_{\lambda}=(1-\alpha) R U L_{\lambda}^{*}\right)$, the component is stopped as this time is smaller than $h \Delta t$.

- With probability $\left(1-P_{\lambda}^{\alpha}\right) F N_{\lambda}, \mathrm{Y}_{\lambda}$ will be above $(1+\alpha) R U L_{\lambda}^{*}$. In this situation, we will not stop the component.

- With probability $1-F N_{\lambda}-F P_{\lambda}$ we are in the situation in which the predicted $R U L$ value is between $\left[\alpha_{\lambda}^{-} \alpha_{\lambda}^{+}\right]$. To be conservative, we assume that also in this case we do not remove the component from operation.

To conclude, a conservative estimation of the stop probability in the time window $\left[T_{p r}+k^{*} \Delta t, T_{p r}+h^{*} \Delta t\right]$ is $\left(1-P_{\lambda}^{\alpha}\right) F P_{\lambda} \simeq\left(1-p_{\lambda}^{\alpha}\right) f p_{\lambda}$.

Fig. 1 briefly summarizes the considerations proposed above. Two different trials of the same PHM-equipped component are plotted over time, which are indicated with superscript 1 (continuous line) and 2 (dashed line). $T_{p r}^{1}$ and $T_{p r}^{2}$ indicate the corresponding first prediction times, whereas $k^{* 1}$ and $k^{* 2}$ represent the first time instants where the system can be stopped with probabilities $\left(1-P_{\lambda}^{\alpha}\right) F P_{\lambda} ; h^{* 1}$ and $h^{* 2}$ are the first time indexes from which the system is stopped with probability $P_{\frac{k}{N}}^{\alpha}+\left(1-P_{\frac{k}{N}}^{\alpha}\right)\left(1-F N_{\frac{k}{N}}\right)$. Finally, $T_{f}^{1}$ and $T_{f}^{2}$ represent the last possible prediction instants before failure and are considered as failure times within our framework.

Based on the considerations above, it is now possible to compute the unreliability $U(t)$ at time $t$, which is here defined as the probability of reaching the failure threshold before $t$ :

$$
\begin{aligned}
U(t)= & \mathbb{P}\left(T_{f} \leq t \cap \text { system not stopped before } t ; \alpha, \beta, \Delta t, h, f n, f p, p_{\lambda}^{\alpha}\right) \\
= & \mathbb{P}\left(T_{f} \leq t \mid \text { system not stopped before } t ; \alpha, \beta, \Delta t, h, f n, f p, p_{\lambda}^{\alpha}\right) \\
& \times \mathbb{P}\left(\text { system not stopped before } t ; \alpha, \beta, \Delta t, h, f n, f p, p_{\lambda}^{\alpha}\right)
\end{aligned}
$$

where $\alpha, \beta, \Delta t, h, f n, f p, p_{\lambda}^{\alpha}$ explicitly indicate the dependence of the unreliability value on the parameters determining the performance of the PHM system.

Notice that there are several definitions of reliability [21]. Differently from the 'traditional' definitions, in which the unreliability is the $\mathrm{CDF}$ of the failure time and, thus, it tends to one as $t$ increases (i.e., the component will always fail, $[21,22])$, in this case we are compelled to consider

$\lim _{t \rightarrow \infty} U(t)=\mathbb{P}\left(\right.$ system not stopped before $t ; \alpha, \beta, \Delta t, h$, fn, f $\left.p, p_{\lambda}^{\alpha}\right) \leq 1$

The difference is due to the fact that if the component is removed from operation before failure, then its failure time will no-longer exist and

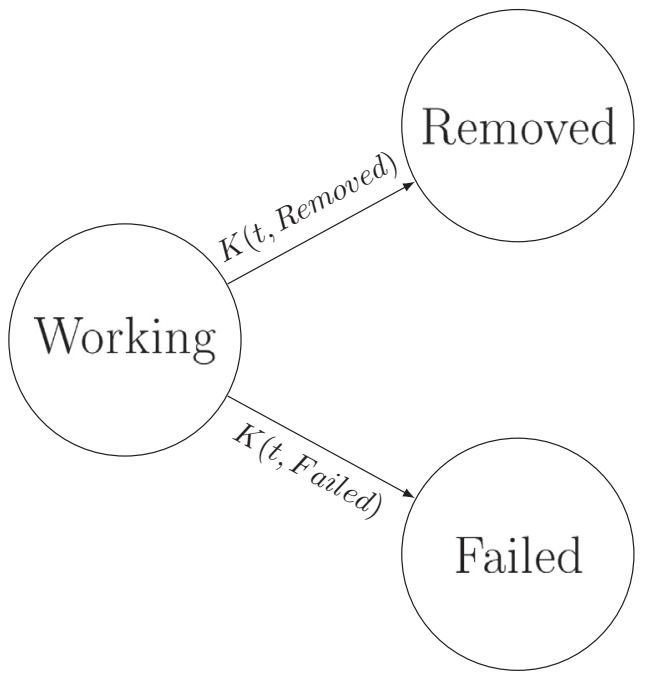

Fig. 4. Three-state system.

the 'traditional' definitions are no longer applicable. That is, the PHMequipped component can be framed as a three-state system, the possible states being: Working, Failed and Removed (Fig. 4), in which $U(t)$ represents the probability of having a transition from Working to Failed before time $t$. According to this view, we derive $U(t)$ from the probabilistic transport kernel $K\left(t\right.$, Failed $\left.\mid t^{\prime}, s^{\prime}\right)$, which is defined as the probability density that the component makes the next transition between $t$ and $t+d t$ toward state Failed [23], provided that the previous transition has occurred at time $t^{\prime}$ and hat the system had entered in state $s^{\prime}$. However, in our case we assume that the component always starts at $t=0$ in state Working. For this, we will indicate the kernel as $K(t$, Failed $)$, without the conditioning event.

To calculate $K(t$, Failed $)$, we first first calculate the failure transportation kernel given a realization $\delta$ from $f_{D T D}$ :

$$
\begin{aligned}
& K\left(t, \text { Failed } \mid \delta ; \alpha, \beta, \Delta t, h, f n, f p, p_{\lambda}^{\alpha}\right) \\
&=\int_{t-\delta}^{t} f_{T_{d}}(\tau) f_{T_{\phi}}(t-\tau) d \tau+\int_{0}^{t-\delta} f_{T_{d}}(\tau) f_{T_{\phi}}(t-\tau) \\
& \prod_{k=k^{*}}^{h^{*}-1}\left[1-\left(1-p_{\frac{k}{N}}^{\alpha}\right) f p_{\frac{k}{N}}\right] \prod_{k=h^{*}}^{N-1}\left[\left(1-p_{\frac{k}{N}}^{\alpha}\right) f n_{\frac{k}{N}}\right] d \tau
\end{aligned}
$$

In other words, it is assumed that a failure occurs when one out of the following conditions is satisfied, which are represented by the first and the second addend of Eq. (4), respectively:

1. The component fails before PHM alerts the detection threshold (detection error); this may happen in case the component fails abruptly.

2. PHM correctly detects, with detection delay $\delta$, that the degradation has reached the detection threshold but, then, over-estimates the actual failure time $T_{f}$ (prognostic error); this happens after $T_{p r}+k^{*} \Delta t$ (i.e., the first prediction instant where the stopping decision should be made), with probability $1-\left(1-p_{\frac{k}{N}}^{\alpha}\right) f p_{\frac{k}{N}}$ and with probability $\left(1-p_{\frac{k}{N}}^{\alpha}\right) f n_{\frac{k}{N}}$ from $T_{p r}+h^{*} \Delta t$ on.

To remove the dependence from $\delta$, we integrate Eq. (4) over the distribution of DTD:

$$
\begin{aligned}
& K\left(t, \text { Failed } ; \alpha, \beta, \Delta t, h, f n, f p, p_{\lambda}^{\alpha}\right) \\
& \quad=\int_{0}^{\infty} K\left(t, \text { Failed } \mid \delta ; \alpha, \beta, \Delta t, h, f n, f p, p_{\lambda}^{\alpha}\right) f_{D T D}(\delta) d \delta
\end{aligned}
$$

Generally speaking, the integral of $K(t$, Failed $)$ over the time interval $\left[t_{1}, t_{2}\right]$ gives the probability of failure in that time span [23]. Then, 


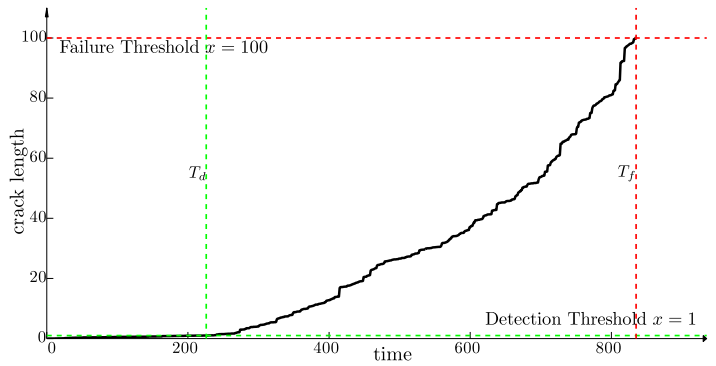

(a) Behaviour in the whole life cycle

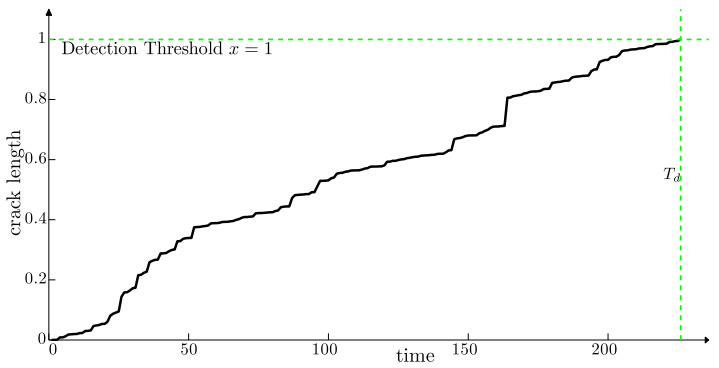

(b) Zoom before first threshold

Fig. 5. Crack propagation process: example

Eq. (5) allows estimating the component unreliability as:

$U(t)=\int_{0}^{t} K\left(\tau\right.$, Failed $\left.; \alpha, \beta, \Delta t, h, f n, f p, p_{\lambda}^{\alpha}\right) d \tau$

Finally, notice that the developed model allows considering also the case where there is no advantage in removing the component from operation the last $h$ instants: in this case, stopping the component in the third region (region 1 in Fig. 3 ) is equivalent to having a failure.

\section{Case study}

In this Section, we illustrate the application of the modeling framework developed to a component affected by fatigue degradation, described by the Paris Erdogan (PE) model ([2,24], Fig. 5):

1. The crack length $x_{i}$ reaches the first threshold, $x=1 \mathrm{~mm}$, according to the following equation:

$x_{i+1}=x_{i}+a \times e^{\omega_{i}^{1}}$

where $a=0.003$ is the growth speed parameter and $\omega_{i}^{1} \sim$ $\mathcal{N}(-0.625,1.5)$ models the uncertainty in the speed values. The uncertainty in the arrival time at $x=1$ is described by pdf $f_{T_{d}}(t)$.

2. The crack length reaches the failure threshold $x=100 \mathrm{~mm}$ according to the following equation:

$x_{i+1}=x_{i}+C \times e^{\omega_{i}^{2}}\left(\eta \sqrt{x_{i}}\right)^{n}$

where $C=0.005$ and $n=1.3$ are parameters related to the component material properties, and are determined by experimental tests; $\eta=1$ is a constant related to the characteristics of the load and the position of the crack and $\omega_{i}^{2} \sim \mathcal{N}(0,1)$ describes the uncertainty in the crack growth speed values. The uncertainty in the arrival time at $x=100$ is described by pdf $f_{T_{f}}(t)$.

The numerical values are taken from [2].

\section{Validation of the reliability model}

The aim of this Section is to validate the reliability model developed in Section 3 by way of the case study presented above. To do this, we carry out the following steps, which are detailed in the next Sections:

- Choose the prognostic and detection algorithms that are assumed to be implemented in the PHM system.

- Estimate the performance values $f p_{\lambda}, f n_{\lambda}$ and $p_{\lambda}^{\alpha}$.

- Estimate the integral in Eq. (6).

- Estimate the unreliability in the 'on-line' setting, in which the crack propagation is simulated together with the selected prognostic and detection algorithms, and with the decisions based on their outcomes as well.

\subsection{Algorithms}

The prognostic algorithm we rely on is Particle Filtering (PF, $[25,26])$, which has been established as the de-facto state of the art in failure prognostics [27]. Briefly, at any time instant PF estimates the pdf of the degradation state of the component (i.e., its crack depth in our case) with a set of weighted particles, which constitute a probability mass function (pmf). When a measure of the crack depth is acquired, such pmf is adjusted in a Bayesian perspective, so that the weights related to particles which are near the acquired data are augmented.

The PF algorithm chosen for our application is the same as that used in [1]; it relies on a simplified approach for predicting the evolution of the crack, which does not give full account to the uncertainty in the particle evolution [1]. Certainly, more refined versions of PF could be considered to improve the prognostic performance, but this is out of the scope of this work: our aim is to check whether the model developed in Section 3 provides conservative estimates of the component reliability for a given set of performance values $f p_{\lambda}, f n_{\lambda}$, and $p_{\lambda}^{\alpha}$, whichever the prognostic algorithm is.

As mentioned in Section 2, our model mainly focuses on prognostics. Thus, we assume that the uncertainty in DTD is already known and it is described by a normal distribution, which for the simulations that follows, is arbitrarily taken to have mean 5 and standard deviation 1 , in arbitrary units. Then, in the simulations, the degradation is detected to reach the detection threshold at a time $T_{p r}$, which is on average 5 time units larger than $T_{d}$ and the variability of this delay is given by the standard deviation of 1 unit. Finally, with respect to the maintenance policy settings, we assume $h=1, \beta=40$ and $\Delta t=30$ in arbitrary units: larger values of $h$ or smaller values of $\beta$ would result in reliability values very close to 1 , which do not allow a fair validation of the proposed modeling framework.

\subsection{Performance estimation}

To estimate the values of the performance metrics $F P_{\lambda}, F N_{\lambda}$ and $P_{\lambda}^{\alpha}$, we implement the following MC procedure:

1. Simulate the crack propagation mechanism to find $T_{f}, T_{d}$, the $N$ prediction instants at every $\Delta t$ time and the corresponding crack lengths. In particular, $T_{p r}$ is obtained by adding a sample from $\mathcal{N}(5,1)$ to $T_{d}$, whereas $T_{f}=R U L^{*}$ at $\lambda=0$. The gathered values of $T_{f}$ and $T_{d}$ are also used to derive $f_{T_{\phi}}$ and $f_{T_{d}}$, respectively, at step 3.

2. At every prediction instant $t_{\lambda}, \lambda=\frac{t_{\lambda}-T_{p r}}{T_{f}-T_{p r}}$, run the PF algorithm to estimate the current crack length and the pdf $f_{R_{\lambda}}$ of the predicted RUL $R_{\lambda}$. On this basis, use Eqs. (1)-(3) to calculate the values of $\Phi P_{\lambda}, \Phi N_{\lambda}$ and $\Pi_{\lambda}^{\alpha}$ using $f_{R_{\lambda}}$ and $R U L_{\lambda}^{*}=T_{f}-t_{\lambda}$. In this respect, Fig. 6, shows the histograms of $\frac{k^{*}}{N}$ and $\frac{h^{*}}{N}$ over $\lambda$ as derived from the simulation of 15,000 Monte Carlo trials of crack degradation: it can be seen that in almost $90 \%$ of the trials, $\frac{k^{*}}{N} \geq 0.9$ and 


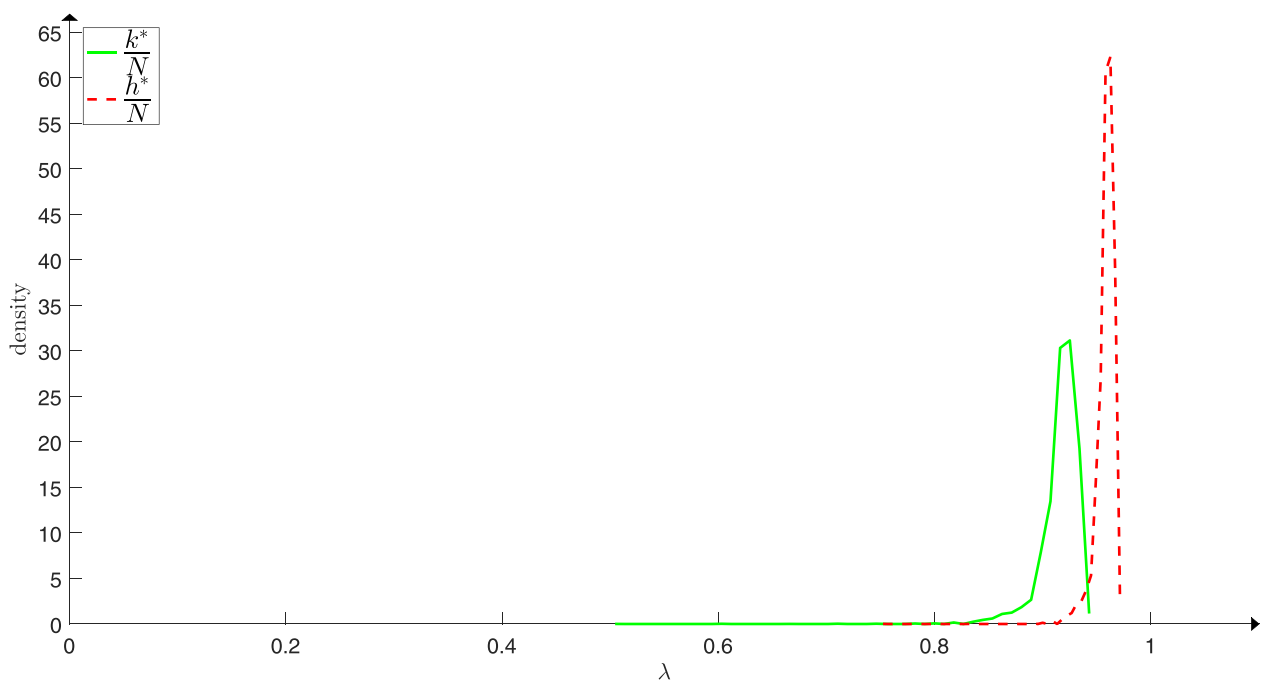

Fig. 6. $\frac{k^{*}}{N}$ and $\frac{h^{*}}{N}$ vs $\lambda$.

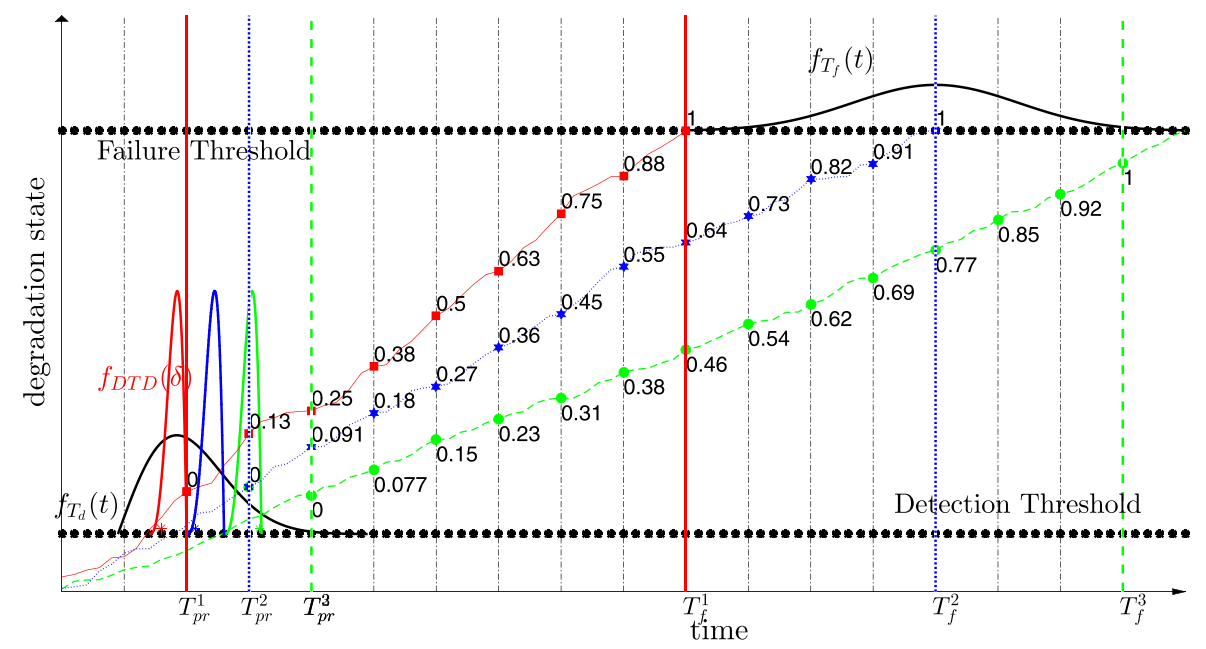

Fig. 7. Example of degradation evolutions and computation of $\lambda$ related to prediction instants.

$\frac{h^{*}}{N} \geq 0.95$. This implies that we can avoid calculating $\Phi P_{\lambda}, \Phi N_{\lambda}$, and $\Pi_{\lambda}^{\alpha}$ for all values of $\lambda$; rather, we can reduce the range of interest at $\lambda>\lambda^{*}$, for some appropriate value of $\lambda^{*}$.

3. Once steps 1 and 2 are simulated a large number of times and the corresponding values of $\Phi P_{\lambda}, \Phi N_{\lambda}$, and $\Pi_{\lambda}^{\alpha}$ are collected, divide $\left[\lambda^{*}, 1\right)$ in $I$ intervals of the same length $\left[\lambda_{i}, \lambda_{i+1}\right), \lambda_{0}=\lambda^{*}, \lambda_{I}=1$; I should be small enough that intervals $\left[\lambda_{i}, \lambda_{i+1}\right)$ do not contain multiple prediction instants of the same MC trial. Derive also $f_{T_{d}}$ and $f_{T_{\phi}}$.

4. For each interval $\left[\lambda_{i}, \lambda_{i+1}\right)$, compute the average of the values of $\Phi P_{\lambda}, \Phi N_{\lambda}$, and $\Pi_{\lambda}^{\alpha}$ gathered at the time instant $\lambda \in\left[\lambda_{i}, \lambda_{i+1}\right)$; this provides the estimates $f p_{\lambda}, f n_{\lambda}$, and $p_{\lambda}^{\alpha}$, which are step-wise functions over the identified $I$ intervals.

Fig. 7 and 8 provide an example of the described procedure for $3 \mathrm{MC}$ trials, in which $\lambda^{*}=0.1$. The degradation paths are simulated over time (Fig. 7) and the corresponding values of interest are collected. Fig. 7 also reports for every degradation path the $\lambda$ values corresponding to the prediction instants, which depend on the duration of the component life. Then, Fig. 8 partitions the interval $[0.1 ; 1)$ in intervals of length 0.06 , which contain at most one prediction instant of the same trial. In this respect, a simple rule to select the maximum $\lambda$ interval length is to select the maximum number $N_{m}$ of prediction instants in a single trial; then, the maximum $\lambda$ interval length is $\frac{1}{N_{m}}$.

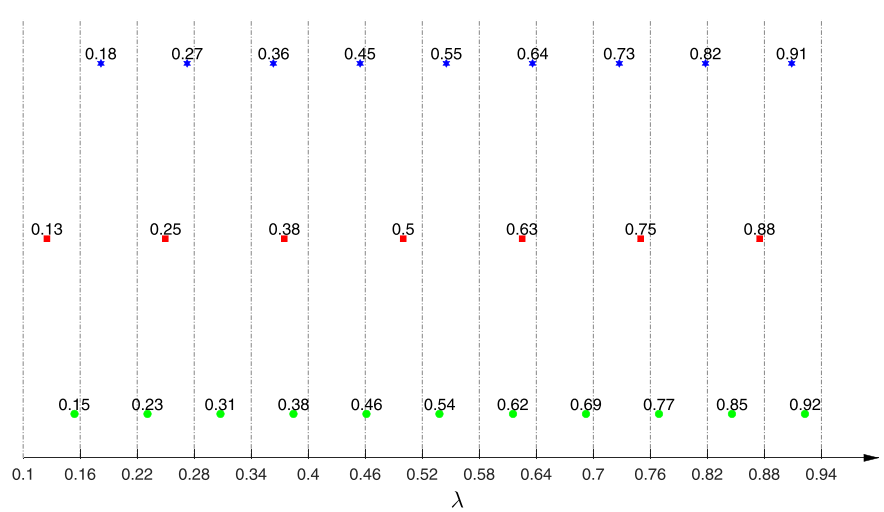

Fig. 8. Computation of the performance metric values of Fig. 7.

Fig. 9 shows the results of the procedure detailed above for the case study illustrated in Section 4, starting from $\lambda \geq \lambda^{*}=0.45$. In particular, two different length values of the $\left[\lambda_{i}, \lambda_{i+1}\right)$ intervals have been considered: 0.05 (Fig. 9a) and 0.005 (Fig. 9b). In both cases, we checked that every interval contains at most one prediction instant of the same trial, although for some simulated trial some intervals do not contain any prediction (see Fig. 8). This causes the noisy behavior of the metrics 


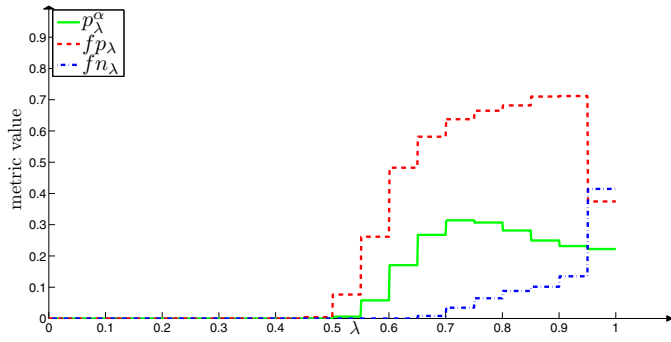

(a) Interval length 0.05

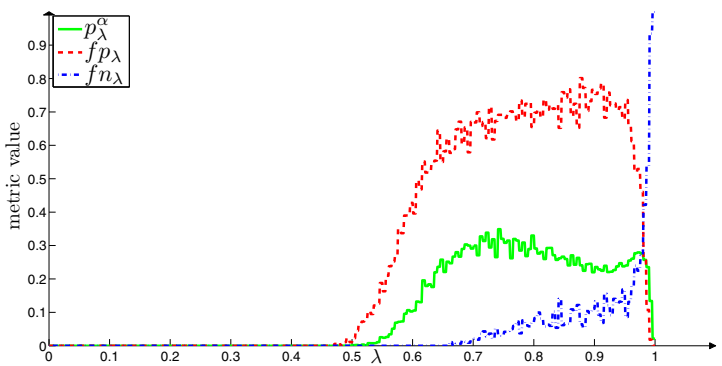

(b) Interval length 0.005

Fig. 9. Experimental metrics values.

in Fig. 9b, as the narrower the interval $\left[\lambda_{i}, \lambda_{i+1}\right)$, the smaller the corresponding number of gathered values of $\Phi P_{\lambda}, \Phi N_{\lambda}$ and $\Pi_{\lambda}^{\alpha}$ over the Monte Carlo trials, the larger the MC error affecting the averaged values.

From the analysis of Fig. 9, we can notice that both the $f p$ and $f n$ values increase over $\lambda$, except for $\lambda \geq 0.95$. This is due to fact that when $\lambda \simeq 1, R U L^{*}=N(1-\lambda) \Delta t \simeq 0$; hence, on one side the chances of having $\phi p=1$ reduces, whereas on the other side there is no possibility to have $\phi n=0$. This entails that $f p_{\lambda}$ and $f n_{\lambda}$ tend to converge to 0 and 1 , respectively as $\lambda \rightarrow 1$. Moreover, $f p$ is always larger than $f n$, except when $\lambda$ $\geq 0.95$. This can be easily explained remembering that we are tracing a percentile of the RUL, which favors the false positive alarms. For the same reason, $p_{\lambda}^{\alpha}$ tends to converge to 0 in the last part of the component life cycle: when the component is approaching its failure time, the RUL estimations become more precise; then, tracking a percentile instead of the RUL median introduces a bias that impacts on the prediction accuracy (see Fig. 2). Notice that this behavior does not contradict the presented model: predictions done at failure time are not considered, as the component is always assumed to fail at $N \Delta t$, which implies that the largest possible value of $\lambda=\frac{(N-1)}{N}<1$.

\subsection{Component unreliability estimation}

To estimate the component unreliability based on Eqs. (4)-(6), the following procedure, derived from [23], has been implemented:

- Divide the time horizon in $J$ time intervals of length $\Delta t$, $\left[0, t_{1}\right),\left[t_{1}, t_{2}\right), \ldots,\left[t_{J-1}, t_{J}\right]$, and associate a counter to every interval, whose initial value is set to 0 .

- Sample $\delta \sim f_{D T D}$; this way, we can estimate the Kernel in Eq. (4), which is conditional on DTD.

- Compute the first addendum of Eq. (4) by Monte Carlo, evaluating the integral corresponding to the undetected failure probability: for each failure time $t_{j}$, we sample $T_{p r}$ from $V\left(t_{j}-\delta, t_{j}\right)$, i.e., a uniform distribution between $t_{j}-\delta$ and $t_{j}$ (see forced simulation in [23]).

- Compute the second integral of Eq. (4), similarly to the previous one except that $T_{p r}$ must be sampled from $\mathcal{V}\left(0, t_{j}-\delta\right)$. Then, $k^{*}, h^{*}$ and $N$ are computed, and the values of the performance metrics obtained are used to complete Eq. (4).

- Estimate $k\left(t_{j}\right.$, Failed $)$ of the integral in Eq. (5) by applying MC method [23].

- Estimate the unreliability at time $t_{j}$ (Eq. (6)), by summing all the failure contributions on the right of $t_{j}$ :

$u\left(t_{j}\right) \simeq \Delta t \sum_{i=1}^{j}\left[k\left(t_{j}\right.\right.$, Failed $\left.)\right] \quad j=1, \ldots, J$

\subsection{Estimation of the 'on-line' unreliability}

The online unreliability is estimated through the MC procedure developed in [1]. Briefly, the time horizon is partitioned in time-channels

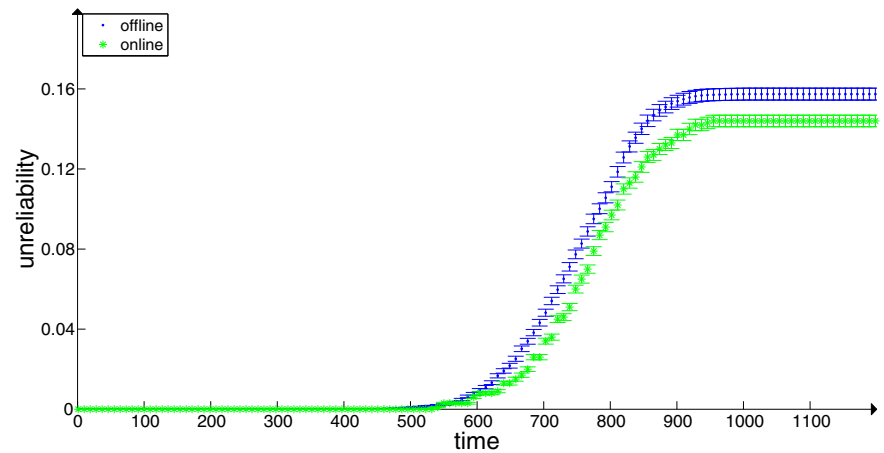

Fig. 10. Comparison between 'on-line' and 'off-line' unreliabilities.

of length $\Delta t$ units of time. The crack growth process is simulated over time together with DTD to compute $T_{d}$. If $T_{p r} \geq T_{f}$, the unreliability counters associated to the channels from $T_{f}$ to the end of the time window are set to 1 ; otherwise, the empirical pdf $f_{R_{\lambda}}$ is estimated every $\Delta t$ units of time by means of the Particle Filtering. Then, at each prediction time $t_{\lambda}$, if the predicted $\beta$ th percentile of $f_{R_{\lambda}}$ is before the next $h$ th inspection time, then the component is removed from operation, otherwise it continues to work. The trial simulation continues until either the component fails or is removed from operation: in the former case, the unreliability counters associated to the channels from $T_{f}$ to the end of the time window are set to 1 ; otherwise they are set to 0 . Finally, the online unreliability at every $\Delta t$ is estimated as the average over many MC simulation trials of the accumulated counter values. As mentioned before, we expect that the offline unreliability curve is always above the online one, as we have built a model which under-estimates the safety benefit of a PHM system.

\subsection{Results}

Fig. 10 shows the two unreliability curves obtained using the two methods described above. The bars in Fig. 10 represent the 68\% twosided confidence interval of the MC simulation error, both in the on-line and off-line setting. From the analysis of the Figure, it seems fair to say that the proposed reliability model is accurate, as the two curves are close to each other. Notice that the difference between the two curves increases with time, meaning that there are no prediction instants at which our model over-estimates the component stopping probability.

\section{Conclusion}

In this work, we have presented a novel general framework to compute the reliability of a PHM-equipped component. The modeling framework proposed applies to safety critical components and risk-averse contexts (e.g., applications of the nuclear, aerospace, oil and gas industries), 
where the main concern is to prevent the component from unexpected failures.

The proposed framework is based on time-variant prognostic metrics of literature ( $F P, F N$ and $P_{\lambda}^{\alpha}$ ) and allows deriving a conservative, analytic model to estimate the failure probability. Application to a mechanical component subject to fatigue degradation has shown that the reliability estimate is close to that obtained from real-time simulation and always under-estimating it.

Further research work is ongoing to investigate the application of the developed reliability model to other engineering applications and to propose further improvements in the reliability model, e.g., for relaxing some conservative assumptions or approximations.

In particular, future research work will focus on the extension of the proposed approach to multi-component systems [28,29]. This will require to encode the diagnostic performance metrics in the proposed reliability model, so as to give due account to the possible system failure paths corresponding to the different degradation evolutions of its components.

\section{Acknowledgment}

The participation of Enrico Zio to this research is partially supported by the China NSFC under grant number 71231001 .

\section{References}

[1] Zio E, Compare M. Evaluating maintenance policies by quantitative modeling and analysis. Reliabil Eng Syst Saf 2013;109:53-65

[2] Zio E. Prognostics and health management of industrial equipment. Diagn Prognostics Eng Syst 2012:333-56.

[3] oes MMS, Gomes JM, Yasin CF. A literature review of maintenance performance measurement: a conceptual framework and directions for future research. J Qual Maint Eng 2011:116-37.

[4] Wang H. A survey of maintenance policies of deteriorating systems. european journal of operational research. Eur J Oper Res 2002;139(3):469-89.

[5] Zio E. Challenges and opportunities in reliability engineering: the big KID (knowledge, information and data). IEEE Trans Reliabil 2016;65(4):1769-82.

[6] Louhichi R., Liu J., Zio E.. Evaluating the economic performance of failure prognostic methods based on return of investment and risk index. submitted for publication.

[7] Youn BD, Hu C, Wang P. Resilience-driven system design of complex engineered systems. J Mech Des 2011;133(10). Art. no. 101011.

[8] Dzakowic JE, Valentine GS. Advanced techniques for the verification and validation of prognostics \& health management capabilities. Machinery failure prevention technologies (MFPT 60), Virginia Beach, VA; 2007.
[9] Goodman L, Wood S, Turner A. Return-on-Investment (ROI) for electronic prognostics in mil/aero systems. IEEE, Autotestcon Orlando, FL; 2005.

[10] Wood S, Goodman D. Return-on-Investment (ROI) for electronic prognostics in high reliability telecom applications. Annual international telecommunications energy conference; 2006.

[11] Compare M, Bellani L, Zio E. Availability model of a PHM-equipped component. IEEE, transactions on reliability; 2017. doi:10.1109/TR.2017.2669400.

[12] Compare M, Baraldi P, Turati P, Zio E. Interacting multiple-models, state augmented particle filtering for fault diagnostics. Probabilistic Eng Mech 2015;40:12-24.

[13] Venkatasubramanian V, Rengaswamy R, Yin K, Kavuri SN. A review of process fault detection and diagnosis. Part I. Quantitative model-based methods. Comput Chem Eng 2003;27(3):293-311.

[14] Saxena A, Celaya J, Saha B, Saha S, Goebel K. Metrics for offline evaluation of prognostic performance. Int J Prognostics Health Manage 11 2010:4-23.

[15] Saxena A, Celaya J, Saha B, Saha S, Goebel K, Schwabacher M, et al. Metrics for evaluating performance of prognostic techniques. In: IEEE, International conference on prognostics and health management; 2008. p. 1-17.

[16] Sankararaman S, Daigle MJ, Goebel K. Uncertainty quantification in remaining useful life prediction using first-order reliability methods. IEEE Trans Reliabil 2014;63(2):603-19.

[17] Bechhoefer E, Bernhard AP. A generalized process for optimal threshold setting in HUMS. Aerospace conference. IEEE; 2007. p. 1-9.

[18] Samuel PD, Pines DJ. A review of vibration-based techniques for helicopter transmission diagnostics. J Sound Vibrat 2005;282(1):475-508.

[19] Giurgiutiu V, Cuc A, Goodman P. Review of vibration-based helicopters health and usage monitoring methods. South Carolina University, Columbia Department of Mechanical Engineering; 2001.

[20] Cannarile F, Baraldi P, Compare M, Borghi D, Capelli L, Cocconcelli M, et al. An unsupervised clustering method for assessing the degradation state of cutting tools used in the packaging industry. In: Proceedings of European safety and relaibility conference, ESREL; 2017. in press.

[21] Kapur KC, Pecht M. Reliability engineering. John Wiley \& Sons; 2014.

[22] Rausand M, Hoyland A. System reliability theory. Models, statistical methods, and applications. second ed. John Wiley \& Sons, Hoboken, New Jersey; 2004.

[23] Zio E. The Monte Carlo simulation method for system reliability and risk analysis. Springer; 2013.

[24] Paris P, Erdogan F. A critical analysis of crack propagation laws. J Basic Eng 1963;85(4):528-33.

[25] Arulampalam MS, Maskell S, Gordon N, Clapp T. A tutorial on particle filters for online nonlinear/non-gaussian Bayesian tracking. IEEE Trans Signal Process 2002;50(2):174-8.

[26] Doucet A, de Freitas JFG, Gordon NJ. Sequential Monte Carlo methods in practice. New York: Springer-Verlag; 2001.

[27] Orchard ME, Tang L, Goebel K, Vachtsevanos G. A novel RSPF approach to prediction of high-risk, low-probability failure events. In: Proceedings of the annual conference of the prognostics and health management society 2009, San Diego, CA; 2009.

[28] Daigle M, Bregon A, Roychoudhury I. A distributed approach to system-level prognostics. National aeronautics and space administration MOFFETT field CA ames research center; 2012

[29] Khorasgani H, Biswas G, Sankararaman S. Methodologies for system-level remaining useful life prediction. Reliabil Eng Syst Saf 2016;154:8-18. 